\title{
L-Phenylalanine Transport in Saccharomyces cerevisiae: Participation of GAP1, BAP2, and AGP1
}

\author{
Daniel A. Sáenz, Mónica S. Chianelli, and Carlos A. Stella \\ Departamento de Bioquímica Humana, Facultad de Medicina, Paraguay 2155, 1121 Buenos Aires, Argentina \\ Correspondence should be addressed to Carlos A. Stella; cstella@fmed.uba.ar
}

Received 4 October 2013; Revised 1 December 2013; Accepted 30 December 2013; Published 20 February 2014

Academic Editor: Dorothy Gietzen

Copyright (C) 2014 Daniel A. Sáenz et al. This is an open access article distributed under the Creative Commons Attribution License, which permits unrestricted use, distribution, and reproduction in any medium, provided the original work is properly cited.

\begin{abstract}
We focused on the participation of GAP1, BAP2, and AGP1 in L-phenylalanine transport in yeast. In order to study the physiological functions of GAP1, BAP2, and AGP1 in L-phenylalanine transport, we examined the kinetics, substrate specificity, and regulation of these systems, employing isogenic haploid strains with the respective genes disrupted individually and in combination. During the characterization of phenylalanine transport, we noted important regulatory phenomena associated with these systems. Our results show that Agplp is the major transporter of the phenylalanine in a gapl strain growing in synthetic media with leucine present as an inducer. In a wild type strain grown in the presence of leucine, when ammonium ion was the nitrogen source, Bap2p is the principal phenylalanine carrier.
\end{abstract}

\section{Introduction}

Amino acid transport across the Saccharomyces cerevisiae plasma membrane is an active and highly regulated process mediated by transmembrane proteins called permeases.Based on their amino acid sequences, these permeases can be grouped into an amino acid permease family (AAP) that includes 18 members of proteins [1-4].

Amino acid permeases display different specificities and capacities that allow extracellular amino acids to be used either as a general nitrogen source or as building blocks in protein synthesis.

The high-capacity permeases Agplp, Gaplp, and Put $4 p$ are expressed under conditions where amino acids are needed as a source of nitrogen to support growth and are preferentially active in cells growing on poor nitrogen sources, such as allantoin, urea, or proline. When a good nitrogen source such as glutamine, asparagine, or ammonium ions is present, utilization of the poorer nitrogen sources is greatly diminished. This general phenomenon is the result of at least two different regulatory mechanisms: nitrogen catabolic repression (NCR), which affects the synthesis of these permeases [5-8], and nitrogen catabolic inactivation (NCI), which downregulates the activity of several permeases that import these poor nitrogen sources.
Although the sequences of the 18 AAP genes are known and the corresponding amino acid sequences deduced, the kinetic properties of many of the more specific permeases have not been described.

It has been shown that L-phenylalanine is required for S. cerevisiae optimal growth on synthetic media $[9,10]$. However, only limited information concerning the specific permeases involved in the L-phenylalanine transport has been published. For years, it was believed that phenylalanine is transported primarily or exclusively by the general amino acid permease [11]. Although several investigators have observed effects of certain mutations on the transport of phenylalanine [12-14], no detailed study of phenylalanine transport has been made [14], nor has a phenylalaninespecific permease been defined [12]. In a strain disrupted in $G A P 1$, additional disruption of $A G P 1$ greatly reduced growth of yeast on medium containing $1.0 \mathrm{mM}$ phenylalanine as the sole nitrogen source [13]. Although Regenberg et al. [14] observed phenylalanine uptake in a gaplssyl strain overexpressing BAP2, Grauslund et al. [15] observed no change in aromatic amino acid uptake under different conditions.

In the present study, we focused on the participation of GAP1 [16], BAP2 [15, 17], and AGP1 [13, 18] in L-phenylalanine transport. The Bap2p and Agplp permeases belong to a subset of more related AAPs (Cluster I) [3] that also includes Gnplp 
[19], Bap3p [20-22], Tatlp, and Tat2p [23, 24]. The expression of genes encoding the Cluster I permeases is induced by the presence of amino acid in the growth media and requires SSY1, PTR3, and SSY5 gene-products [4, 12, 13, 21, 25]. These genes are essential components of a signalling pathway (SPS pathway) that activates transcription of multiple permease genes.

Schreve et. al [18] identified Agplp as an asparagine and glutamine permease. It was further suggested that Agplp can act as a low-affinity, broad-substrate-range permease that is involved in the uptake of amino acids for catabolism. Subsequently, Iraqui et al. [13] showed that Agplp could transport most neutral amino acids, including phenylalanine with $K_{T}=$ $0.6 \mathrm{mM}$. Regenberg et al. [14] employed overexpression and deletion to show that $B A P 2, A G P 1, B A P 3$, and TAT2 could be involved in phenylalanine uptake. All of these permeases have previously been identified as transporters for other amino acids.

In order to study the physiological functions of GAP1, $B A P 2$, and $A G P 1$ in L-phenylalanine transport, we examined the kinetics, substrate specificity, and regulation of these systems, employing isogenic haploid strains with the respective genes disrupted individually and in combination. During the characterization of phenylalanine transport we noted important regulatory phenomena associated with these systems. Our results show that Agplp is the major transporter of the phenylalanine in a gap1 strain growing in MA or MP media with leucine present as an inducer. In a wild type strain grown in the presence of leucine in MA medium, Bap2p is the principal phenylalanine carrier.

\section{Materials and Methods}

2.1. Strains and Media. The Saccharomyces cerevisiae strains used in this study are all isogenic with the "wild type" parental strain Y294, MAT a ura3-52 leu 2-3,112 his3- $\Delta 1$ trp1-289 [17] except for the mutations mentioned; Y294 $\Delta$ bap2 with bap2::URA3; Y294 gap1 with gap1::LEU2; Y294 $\Delta$ bap2 2 gap1 with bap2::URA3 and gap1::LEU2; [17]; Y294 $\triangle$ agp1 with agp1::URA3, and Y294Aagp1 $\Delta$ gap1 with agp1::HIS3 and gap1::LEU2. The parental strain and all the disruption strains were the generous gift of Garrett.

For general maintenance of yeast cultures, YPD medium (1\% yeast extract (Difco), 2\% peptone (Difco), and 2\% Dglucose) was used. Minimal ammonium (MA) medium contained per liter: $1.7 \mathrm{~g}$ Yeast Nitrogen Base without ammonium sulfate and amino acids (Difco), $5 \mathrm{~g}$ ammonium sulfate, and $2 \mathrm{~g}$ D-glucose. MP medium, and MG or MAA medium were similar to MA except that $1 \mathrm{~g}$ of L-proline, $10 \mathrm{mM}$ of Lglutamine, or $1 \mathrm{mM}$ of an L-amino acid was used as sole nitrogen source. When solid media were required, $2 \%(\mathrm{w} / \mathrm{v})$ Bacto Agar (Difco) was included. All minimal media contained the following supplements unless indicated otherwise: uracil (20 mg/L), L-histidine $(20 \mathrm{mg} / \mathrm{L})$, L-tryptophan $(20 \mathrm{mg} / \mathrm{L})$, and L-leucine $(30 \mathrm{mg} / \mathrm{L}=0.23 \mathrm{mM})$. Assays for resistance to toxic analogues were carried out on plates of MA-leu (MA medium without L-leucine). Concentrations of analogues used were as follows: $15 \mu \mathrm{g} / \mathrm{mL} \quad \beta$ (2-thienyl)-DL-alanine
(Sigma Chemical Co.); $9 \mu \mathrm{g} / \mathrm{mL}$ 5,5,5-trifluoro-DL-leucine (Fairfield Chemical Co.).

2.2. Amino Acid Uptake. Two hundred microliters from an overnight culture was used to inoculate $50 \mathrm{~mL}$ of culture medium as indicated in each case and grown to a cell density corresponding to an $\mathrm{OD}_{570}$ of 1.0 to 2.0 at $30^{\circ} \mathrm{C}$. The cells were harvested, washed twice with $50 \mathrm{~mL}$ of distilled water, and resuspended in distilled water at $\mathrm{OD}_{570}$ of 4.5. The uptake measurements were performed as described by Chianelli et al. [26]. The reaction mixture containing $0.5 \mathrm{mg}$ cell dry weight $\mathrm{mL}^{-1}$ in $20 \mathrm{mM}$ potassium phthalate buffer ( $\mathrm{pH} 4.5$ ) was incubated at $30^{\circ} \mathrm{C}$ with shaking. The reaction was initiated by the addition of $0.1-0.3 \mu \mathrm{ci}$ of $\mathrm{L}-\left[{ }^{14} \mathrm{C}\right]$-phenylalanine at a final concentration of $20 \mu \mathrm{M}$ or $1.0 \mathrm{mM}$. Samples of $0.2 \mathrm{~mL}$ were withdrawn at $0(7 \mathrm{sec}), 2$, and $4 \mathrm{~min}$ in order to determine the initial uptake rate. Uptake saturation kinetics were measured at phenylalanine concentrations between 0.005 and $2.0 \mathrm{mM}$ (specific activity between 0.25 and $10.00 \mu \mathrm{ci} / \mu \mathrm{mol}$ ). The initial rates of uptake were estimated from the initial parts of the curves by linear regression and are expressed as $\mu \mathrm{mol} \mathrm{g}^{-1}$ cell dry weight $\min ^{-1}$. All uptake measurements are the average of 2-3 separate experiments. Data were fitted to MichaelisMenten and Lineweaver-Burk plots using the Solver program of Microsoft Excel 5.0 (Microsoft Corp., Bellevue, WA). The phenylalanine uptake data of Y294 $\Delta$ bap2 and Y294 were fitted assuming two and three transport systems, respectively. The Solver program was used to simultaneously fit the Y294 with the parameters obtained from the Y294 $\Delta$ bap2 data for the two permeases and by adjusting the Bap2 parameters to obtain the best fit. All radiolabelled amino acids used were obtained from New England Nuclear. L-amino acids were purchased from Sigma Chemical Co.

\section{Results}

3.1. Disruption of BAP2 in a gap1 Strain Confers Resistance to Toxic Analogues of Leucine and Phenylalanine. An initial indication that Bap2p could transport phenylalanine was obtained by demonstrating that on MA plates a bap2 gap1 strain was resistant to the phenylalanine analogue $\beta$ (2-thienyl) DL-alanine and to the leucine analogue DLtrifluoroleucine [26] (data not shown) when compared to an isogenic gap1 strain. This result confirms the finding that overexpression of BAP2 in gap1ssy1 cells led to substantial phenylalanine uptake [14].

3.2. Disruption of AGP1 in a gap1 Strain Reduces Growth on Branched-Chain and Aromatic Amino Acids. We compared the growth of the gap1, gap1bap2, and gaplagp1 strains on a low concentration $(1.0 \mathrm{mM})$ of several individual amino acids: leucine, isoleucine, phenylalanine, tryptophan, tyrosine, and threonine, each used as the sole nitrogen source. The gaplbap2 mutant displayed no clear growth defect on any of the amino acids. Interestingly, this growth test failed to show any contribution of Bap2p, defined as the major branchedchain amino acid permease in the utilization of leucine or isoleucine as the sole nitrogen source. However, disruption 
TABLE 1: Phenylalanine transport in a Y294 $\Delta$ gapl strain at different leucine concentrations.

\begin{tabular}{lc}
\hline L-leucine $(\mu \mathrm{M})$ & Initial velocity $\left(\mathrm{nmol} \cdot \mathrm{g}^{-1} \cdot \mathrm{min}^{-1}\right)$ \\
\hline 0 & $138 \pm 6$ \\
10 & $142 \pm 3$ \\
50 & $164 \pm 6$ \\
230 & $227 \pm 23$ \\
\hline
\end{tabular}

Cells were grown in MA medium plus all supplements plus leucine at the indicated concentrations.

$\mathrm{L}-\left[{ }^{14} \mathrm{C}\right]$-phenylalanine concentration was $20 \mu \mathrm{M}$.

of the AGP1 gene in the gap1 strain reduced the growth on low concentrations of leucine, isoleucine, phenylalanine, tryptophan, tyrosine, and threonine (data not shown).

Agplp has been reported to be a permease for most neutral amino acids $[13,14]$, a finding which is confirmed by the present results. We must note that the concentration of the amino acid used as the major nitrogen source is five times higher than the concentration of the amino acid used to compensate for the strain auxotrophies.

\subsection{Activation of Phenylalanine Transport by Extracellular} Leucine. Two important factors that influence phenylalanine transport activity in yeast are the presence of amino acids in the growth medium and the existence of a signalling pathway (SPS or ssyl-dependent pathway). This pathway senses external amino acids and transmits a transcriptional activation impulse to multiple AAP genes, including BAP2 and $A G P 1[12,13,21]$.

Because the deletion mutants used in this study contain different combinations of auxotrophic markers for histidine, leucine, and tryptophan, all growth media contained these three amino acids, together with uracil, unless indicated otherwise. The phenylalanine uptake assay is performed in washed cells suspended in FHK buffer. Thus, the amino acids necessary to compensate for auxotrophies are absent and do not exert a competitive effect on the phenylalanine uptake. The concentration of leucine used was $0.23 \mathrm{mM}(30 \mathrm{mg} / \mathrm{mL})$. This leucine concentration was used by Didion et al. [21] to induce transcription of the amino acid permease genes $B A P 2$, $B A P 3$, and TAT1. Forsberg et al. $[4,25]$ used $0.15 \mathrm{mM}$ leucine to activate transcription of several additional permease genes, including $A G P 1$, via the SPS signalling pathway. As shown in Table 1, addition of leucine caused an increase of $64 \%$ in the initial velocity values in phenylalanine uptake in the Y294 gapl strain. Because the medium already contains $0.10 \mathrm{mM}$ tryptophan, a good signalling activator $[4,13,21,25]$, addition of $0.23 \mathrm{mM}$ leucine caused only a small additional effect.

\subsection{Disruption of BAP2 or AGP1 Leads to a Reduced Uptake} of L-Phenylalanine. To investigate directly the involvement of Gaplp, Bap2p, and Agplp in L-phenylalanine transport in S. cerevisiae, we measured phenylalanine uptake into isogenic yeast strains bearing different mutations under various experimental conditions. The uptake rates were determined in cells grown in both MP and MA media and at two
TABLE 2: Initial velocities of L-citrulline uptake.

\begin{tabular}{lcc}
\hline Strains & MP medium & MA medium \\
\hline Y294 & $103 \pm 4$ & $<6$ \\
Y294 $\Delta$ bap2 & $301 \pm 13$ & $<6$ \\
Y294 $\Delta$ agp1 & $189 \pm 8$ & $<6$ \\
Y294 $\Delta$ gap1 & $<6$ & $<6$ \\
\hline
\end{tabular}

Initial velocity expressed in $\mathrm{nmol} \cdot \mathrm{g}^{-1} \cdot \mathrm{min}^{-1} \cdot \mathrm{L}-\left[{ }^{14} \mathrm{C}\right]$-citrulline concentration was $20 \mu \mathrm{M}$.

different substrate concentrations (Figure 1). At low substrate concentrations, uptake occurs by high-affinity permeases, whereas at high substrate concentrations, amino acid uptake occurs predominantly through low-affinity permeases.

For a number of L-amino acids, namely, glycine, alanine, phenylalanine, tryptophan, and tyrosine [11, 27], Gaplp has been claimed to be the principal transporter in S. cerevisiae cells. The data in Figure 1 indicate that in the absence of general amino acid permease activity, transport of phenylalanine may involve Bap2p, Agplp, and at least one additional transport system. The relative contribution of these alternative systems depends both on the type of nitrogen source and the concentration of phenylalanine available.

As seen from Figures 1(a) and 1(b), in Y294 and Y294 Aagp1 strains a minor decrease in phenylalanine uptake is evident in MA medium, as compared to MP medium. This indicates that Gaplp inactivation by ammonium ion has very limited or no effect on phenylalanine uptake. In contrast, a very important difference in the uptake rate was found when phenylalanine uptakes by Y294 $\Delta$ bap2 yeast cells grown in the two different media were compared. As previously reported, the general amino acid permease, Gaplp, is strongly repressed and inactivated in MA medium $[6,16]$. Inactivation of Gaplp by the ammonium ion in MA medium is confirmed by the results shown in Table 2 .

Uptake of L-citrulline, which is transported only by Gaplp, [16] is negligible in wild type and in both the Y294 $\Delta$ bap2 and Y294 $\Delta$ agp1 mutants, when cells were grown in MA medium. In MP medium, however, all three strains exhibit Gaplp activity, as measured by L-citrulline transport. Whereas the phenylalanine uptake by the three strains grown in MP may be attributed largely to uptake by Gaplp, the uptake by cells grown in MA is catalyzed mainly by Bap2p. Disruption of BAP2 reduces high-affinity phenylalanine transport to about $10 \%$ of the wild type level (Figure 1(a)). At high substrate concentration, low-affinity phenylalanine transport in MA-grown cells also depends upon functional $B A P 2$, but to a lesser extent (Figure 1(b)). Deletion of BAP2 reduces high-affinity phenylalanine transport only $61 \%$. The residual uptake is not due to Agplp, since deletion of AGPI has almost no negative, effect irrespective of phenylalanine concentration (Figures 1(a) and 1(b)).

Deletion of GAP1 causes a minor (20\%) decrease in highaffinity phenylalanine uptake in MP-grown cells (Figures $1(a)$ and 1(c)), but an eightfold increase in low-affinity phenylalanine transport was relative to wild type (Figures 1(b) and 1(d)). For MA-grown cells the increase was 4.4-fold. Because the additional deletion of BAP2 (Figure 1(d)) causes 


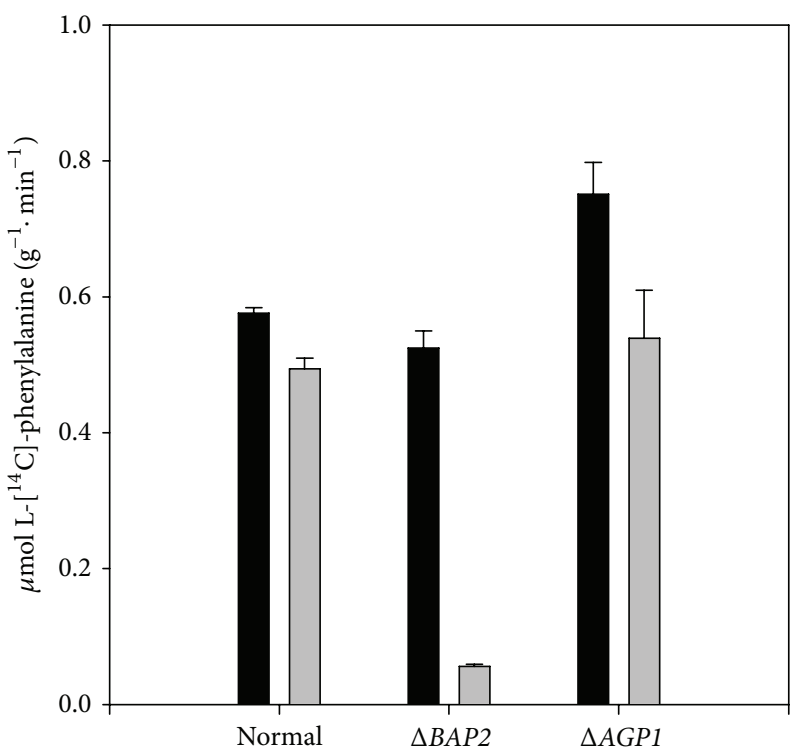

(a)

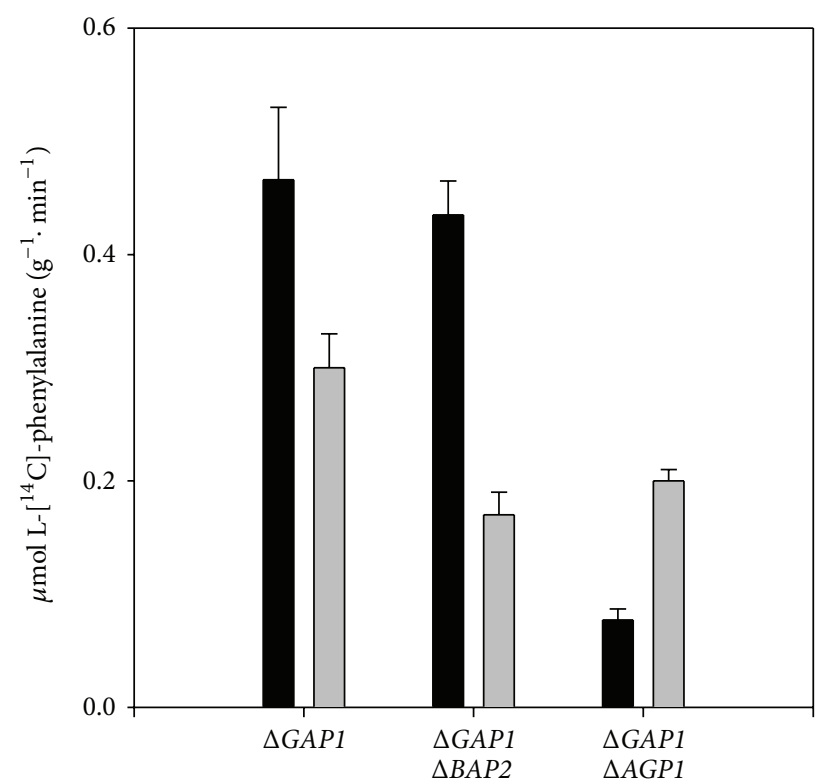

(c)

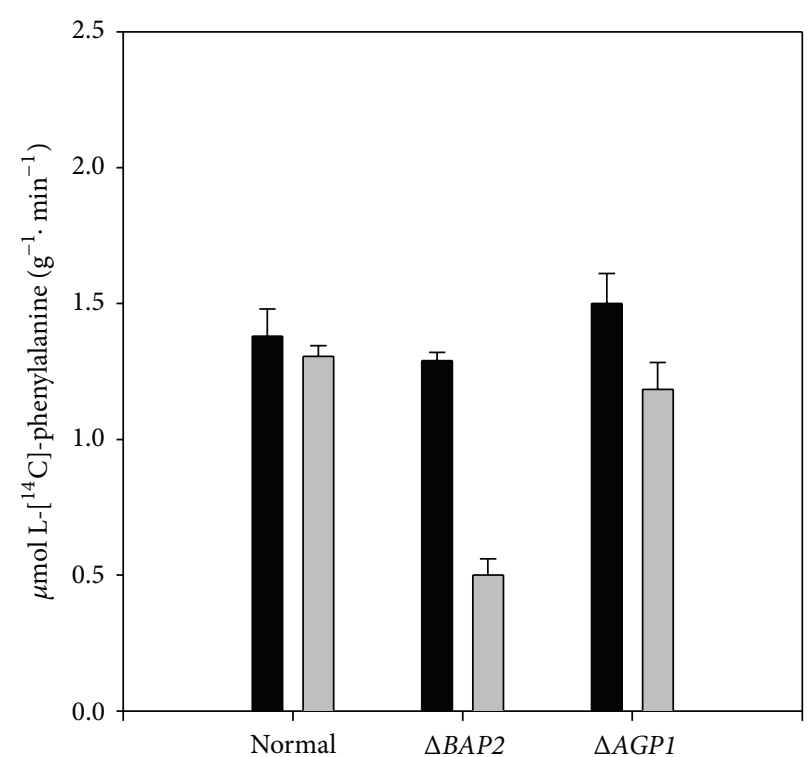

(b)

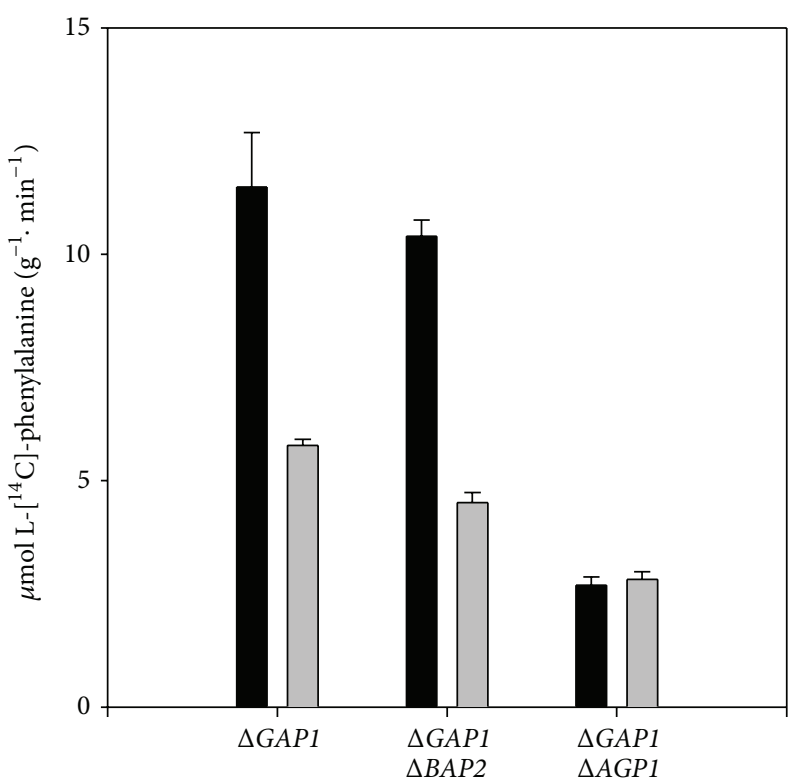

(d)

FIGURE 1: Phenylalanine uptake into Y294 wild type and mutant yeast strains inactivated in GAP1, BAP2, and AGP1. The strains were grown in MP (black bars) or MA (shaded bars) media containing leucine, histidine, tryptophan, and uracil. The L- $\left[{ }^{14} \mathrm{C}\right]$-phenylalanine uptake was assayed as described in Section 2. Phenylalanine uptake was determined at an amino acid concentration of $20 \mu \mathrm{M}$ ((a) and (c)); phenylalanine uptake was determined at amino acid concentration of $1.0 \mathrm{mM}((\mathrm{b})$ and $(\mathrm{d}))$.

only minor changes, it is unlikely that $B A P 2$ is responsible for this high uptake. In contrast, deletion of AGP1 in a gap1 strain causes dramatic decreases in both high- and lowaffinity phenylalanine uptake in MP-grown cells (Figures 1(c) and $1(\mathrm{~d})$ ), indicating that Agplp is acting as the primary phenylalanine carrier in gap1 strains.

In MA-grown cells, Bap2p and Agplp both appear to contribute to both high- and low-affinity phenylalanine uptake (Figures 1(c) and 1(d)). In contrast, when only AGP1 was disrupted, phenylalanine transport was not notably altered in cells grown in either MA or MP medium (Figures 1(a) and 1(b)). Clearly, GAP1 disruption activates AGP1 expression, whereas inactivation/repression of GAP1 does not. (Compare Figures 1(c) and 1(d).)

3.5. Specificity of L-Phenylalanine Transport Systems. It was of interest to determine the specificity of various amino acid permeases for phenylalanine in normal and mutant strains by measuring phenylalanine uptake in the presence of various amino acids and analogues at tenfold higher concentrations. 
TABLE 3: Specificity of phenylalanine uptake in cells grown in $\mathrm{MP}^{\mathrm{a}}$.

\begin{tabular}{|c|c|c|c|}
\hline \multirow{2}{*}{ Unlabelled amino acid added } & \multicolumn{3}{|c|}{ Percentage inhibition } \\
\hline & Y294 & Y294 $\Delta$ bap2 & Y294 $\Delta$ agp1 \\
\hline Leucine & 76 & 75 & 78 \\
\hline Isoleucine & 71 & 71 & 73 \\
\hline Valine & 52 & 71 & 56 \\
\hline Tyrosine & 60 & 81 & 66 \\
\hline Tryptophan & 48 & 86 & 60 \\
\hline Asparagine & 18 & 37 & 19 \\
\hline Citrulline & 15 & 40 & 20 \\
\hline DL-trifluoroleucine & 59 & 42 & 62 \\
\hline
\end{tabular}

${ }^{a}$ High-affinity phenylalanine uptake was measured at 4 min with L- $\left[{ }^{14} \mathrm{C}\right]$-phenylalanine $20 \mu \mathrm{M}$ and the unlabelled amino acids were added at $200 \mu \mathrm{M}$. Control

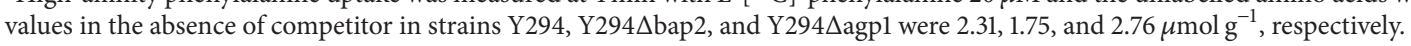

TABLE 4: Specificity of phenylalanine uptake in cells grown in MA medium ${ }^{\mathrm{a}}$.

\begin{tabular}{|c|c|c|c|}
\hline \multirow{2}{*}{$\begin{array}{l}\text { Unlabelled } \\
\text { amino acid added }\end{array}$} & \multicolumn{3}{|c|}{ Percentage inhibition } \\
\hline & Y294 & Y294 $\Delta$ bap2 & Y294 agp1 \\
\hline Leucine & 76 & 34 & 72 \\
\hline Proline & 6 & 0 & 0 \\
\hline DL-TFL ${ }^{\mathrm{b}}$ & 57 & 3 & 48 \\
\hline$\beta-2 \mathrm{TA}^{\mathrm{c}}$ & 71 & 26 & 67 \\
\hline
\end{tabular}

${ }^{a}$ High-affinity phenylalanine uptake was measured at 4 min with L- $\left[{ }^{14} \mathrm{C}\right]-$ phenylalanine $20 \mu \mathrm{M}$ and the unlabelled amino acids were added at $200 \mu \mathrm{M}$. Control values in the absence of competitor in strains Y294, Y294 $\Delta$ bap2, and Y294 $\Delta$ agp1 were 2.29, 0.32 , and $1.91 \mu \mathrm{mol} \mathrm{g}^{-1}$, respectively.

${ }^{\mathrm{b}}$ DL-trifluoroleucine.

${ }^{\mathrm{c}} \beta$-2-thienylalanine.

The results presented in Table 3 were obtained using MP-grown cells in which Gaplp is active in single-deletion strains as well as in wild type. As expected, all amino acids tested, including citrulline, exhibited some competition with phenylalanine. Although the pattern of inhibition was nearly the same in wild type and in the agp1 strain, the bap2 strain exhibited substantially greater inhibition by valine, tyrosine, tryptophan, asparagine and, most notably, by citrulline, and less by DL-trifluoroleucine. These results suggest that elimination of Bap2p activity, but not of Agplp activity, causes the cell to utilize Gaplp to a greater degree. In other words, even in wild type MP-grown cells, Bap2p makes a significant contribution to the high-affinity phenylalanine uptake.

Additional evidence that Bap2p contributes significantly to high-affinity phenylalanine uptake in MA-grown cells is given in Table 4 . Deletion of BAP2 greatly reduces competition by leucine and almost eliminates sensitivity to DLtrifluoroleucine. Nevertheless, the residual phenylalanine transport exhibits some sensitivity to the phenylalanine analogue $\beta$-(2-thenyl) alanine. The results shown in Tables 3 and 4 clearly indicate that (1) the general amino acid permease is the main phenylalanine transporter in the Y294 $\Delta$ bap2 strain grown in MP medium; (2) in wild type and Y294 $\Delta$ agpl, besides Gaplp, another permease, presumably Bap2p, contributes to the transport of phenylalanine in MP medium; (3) in cells grown in MA medium, phenylalanine transport by Bap2p is inhibited specifically by trifluoroleucine (48-57\%).
Under the same experimental conditions the inhibition profiles of phenylalanine uptake are quite different in the gap1 strains (Table 5) as compared to the GAP1 strains (Tables 3 and 4).

The results shown in Table 5 provide further evidence that Agplp is an important transporter in MP-grown cells lacking GAP1. Leucine, tryptophan, and particularly asparagine compete with phenylalanine for transport. Moreover, absence of BAP2 has no effect on the inhibition pattern with these three amino acids. However, when cells are grown in MA, two major differences can be seen. (1) Whereas in Y294 $\Delta$ gap1 and Y294 $\Delta$ bap2 $\Delta$ gapl phenylalanine uptake is inhibited by asparagine, this inhibition is lost in strain Y294 $\Delta$ agpl $\Delta$ gapl. This indicates that phenylalanine transport in MA-grown cells involves Agplp. (2) The participation of Bap2p in highaffinity phenylalanine transport in both Y294 $\Delta$ gapl and Y294 $\triangle$ agpl $\triangle$ gapl strains is supported by the strong inhibition by leucine and DL-trifluoroleucine. When BAP2 is deleted, the trifluoroleucine inhibition almost disappears, and there is a significant decrease in leucine inhibition. The inhibition of phenylalanine uptake by tryptophan in MA-grown cells disrupted in both GAP1 and BAP2, or GAP1 and AGP1, suggests the possible participation of a TAT gene-product. This might also account for the resistance of the Y294 $\Delta$ bap2 $\Delta$ gap1 strain to $\beta$-(2-thienyl) alanine. This might also account for the sensitivity of the Y294 and Y294 $\Delta$ agp1 $\Delta$ gapl strains to $\beta$ (2-thienyl) alanine.

It can be seen in Table 5 that in cells grown in MA medium, the most significant decreases in the inhibition of the phenylalanine uptake were observed with trifluoroleucine in the Y294 $\Delta$ bap2 $\Delta$ gap1 strain and with asparagine in the Y294 $\Delta$ agp1 $\Delta$ gapl strain.

3.6. Kinetic Analysis of L-Phenylalanine Transport by Bap2p and Agplp. In order to study in more detail the involvement of Gaplp, Bap2p, and Agplp in phenylalanine transport we examined the kinetics of phenylalanine uptake by cells of wild type strain Y294 and mutant yeast strains grown in MP and MA media (Table 6). L- $\left[{ }^{14} \mathrm{C}\right]$-phenylalanine uptake was determined at a range of phenylalanine concentrations and results were plotted as Michaelis-Menten or as Eadie-Hofstee graphs (data not shown). 
TABLE 5: Specificity of phenylalanine uptake in gapl cells grown in MP and MA media ${ }^{\mathrm{a}}$.

\begin{tabular}{|c|c|c|c|c|c|c|}
\hline \multirow{3}{*}{ Unlabelled amino acid added } & \multicolumn{6}{|c|}{ Percentage inhibition } \\
\hline & \multicolumn{2}{|c|}{ Y294 dgap1 } & \multicolumn{2}{|c|}{ 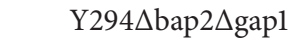 } & \multicolumn{2}{|c|}{ Y294 $\Delta$ agp1 $\Delta$ gap1 } \\
\hline & MP & MA & MP & MA & MP & MA \\
\hline Leucine & 39 & 45 & 34 & 34 & 4 & 51 \\
\hline Tryptophan & 17 & 18 & 18 & 25 & 4 & 23 \\
\hline Asparagine & 45 & 29 & 48 & 35 & 4 & 0 \\
\hline Proline & ND & 8 & ND & 13 & ND & 12 \\
\hline DL-TFL ${ }^{b}$ & ND & 30 & ND & 3 & ND & 37 \\
\hline$\beta-2 \mathrm{TA}^{\mathrm{c}}$ & ND & 43 & ND & 31 & ND & 55 \\
\hline
\end{tabular}

${ }^{a}$ High-affinity phenylalanine uptake was measured at $4 \mathrm{~min}$ with L- $\left[{ }^{[4} \mathrm{C}\right]$-phenylalanine $20 \mu \mathrm{M}$ and the unlabelled amino acids were added at $200 \mu \mathrm{M}$. Control values in the absence of competitor in strains Y294 $\Delta$ gapl, Y294 $\Delta$ bap2 $\Delta$ gapl, and Y294 $\Delta$ agp1 $\Delta$ gapl grown in MP medium were $1.89,1.63$, and 0.44 and for cells grown in MA medium were $1.59,0.98$, and $1.09 \mu \mathrm{mol} \mathrm{g}^{-1}$, respectively.

${ }^{\mathrm{b}}$ DL-trifluoroleucine.

${ }^{\mathrm{c}} \beta$-2-thienylalanine.

TABLE 6: Kinetic parameters of L-phenylalanine transport.

\begin{tabular}{|c|c|c|c|c|c|c|}
\hline Strains & Medium & $K_{T 1}(\mu \mathrm{M})$ & $J_{\max 1}$ & $K_{T 2}(\mathrm{mM})$ & $J_{\max 2}$ & NS \\
\hline \multirow{2}{*}{ Y294 } & MP & $11 \pm 2$ & $0.85 \pm 0.02$ & \multirow{2}{*}{$0.77 \pm 0.3$} & \multirow{2}{*}{$0.46 \pm 0.05$} & $0.42 \pm 0.02$ \\
\hline & MA & $\begin{array}{l}24 \pm 5 \\
67 \pm 26\end{array}$ & $\begin{array}{r}0.86 \pm 0.08 \\
0.21 \pm 0.07\end{array}$ & & & - \\
\hline \multirow{2}{*}{ Y294 $\Delta$ bap2 } & MP & $4.7 \pm 1$ & $0.58 \pm 0.03$ & - & - & $0.46 \pm 0.02$ \\
\hline & MA & $67 \pm 26$ & $0.21 \pm 0.07$ & $0.77 \pm 0.3$ & $0.46 \pm 0.05$ & - \\
\hline \multirow{2}{*}{ Y294 $\Delta$ agp1 } & MP & $11 \pm 1$ & $1.04 \pm 0.04$ & - & - & $0.47 \pm 0.03$ \\
\hline & MA & $26 \pm 2$ & $1.37 \pm 0.02$ & - & - & - \\
\hline \multirow{2}{*}{ Y294 dgap1 } & MP & - & - & $0.75 \pm 0.3$ & $14.3 \pm 2.0$ & $2.97 \pm 1.10$ \\
\hline & MA & 39 & 0.34 & 0.70 & 5.09 & 2.66 \\
\hline \multirow{2}{*}{ Y294 agp1 1 gap1 } & MP & - & - & - & - & 2.91 \\
\hline & MA & 39 & 0.6 & - & - & 2.55 \\
\hline
\end{tabular}

L- $\left[{ }^{14} \mathrm{C}\right]$-phenylalanine uptake was assayed as described in Section 2. Uptake data were plotted as Michaelis-Menten and Lineweaver-Burk plots (data not shown) and the kinetic parameters estimated using the Solver program of Microsoft Excel.

NS: nonspecific $\left(K_{T} \geq 1 \mathrm{M}\right)$.

In cells grown in MP medium, phenylalanine transport is clearly biphasic. The Eadie-Hofstee plots indicate that phenylalanine uptake is mediated by a component of high affinity, presumably Gaplp. The second component either is nonsaturable, or has very low affinity. Computer analysis of these uptake data provided the kinetic parameters given in Table 6. In cells grown in MP medium, as seen from Table 6, there is no significant difference in the kinetic parameters of

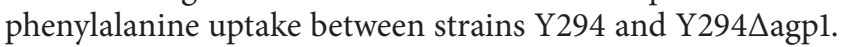
These results indicate that the absence of the Agplp has little or no influence on phenylalanine transport in the presence of the high-affinity permease with $K_{T 1}=11 \mu \mathrm{M}$.

However, in MP-grown cells the disruption of BAP2 (Y294 $\Delta$ bap2 strain) causes a substantial change in the apparent affinity constant for high-affinity phenylalanine uptake

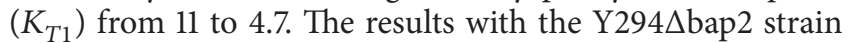
show that although Gaplp is largely responsible for Lphenylalanine uptake, there is apparently some contribution of Bap2p.

Later we analyzed the kinetics of phenylalanine uptake by cells grown in MA medium. For the strains Y294 and Y294 $\Delta$ bap2, the Eadie-Hofstee plots are clearly biphasic, whereas for the strain Y294 $\Delta$ agpl the plot is linear. These results confirm that phenylalanine uptake is mediated by multiple permeases with different affinities and capacities.

The disruption of BAP2 gene specifically results in the absence of the high-affinity phenylalanine uptake component (see Table 6). The apparent $K_{T}$ value of Bap2 p was found to be $24 \mu \mathrm{M}$ and the $J_{\max }$ value was $0.86 \mu \mathrm{mol} / \mathrm{min}$ per $\mathrm{g}$. of dry weight. The phenylalanine uptake kinetics for the strain Y294 $\Delta$ bap2 indicates that there is also a small contribution to phenylalanine uptake by two more permeases in strain Y294 grown in repressing (MA) conditions (Table 6). One of these has relatively high affinity, $K_{T}=67 \mu \mathrm{M}$, while the other has low affinity for phenylalanine $\left(K_{T 2}=0.77 \mathrm{mM}\right)$.

Apparently, when $A G P 1$ is disrupted, only a minor part of phenylalanine uptake is abolished in MA-grown cells. The uptake data for the Y294 $\Delta$ agp1 strain shows the loss of the low-affinity Agplp permease $\left(K_{T}=0.77 \mathrm{mM}\right.$ and $J_{\max }=0.46 \mu \mathrm{mol} / \mathrm{min} / \mathrm{g}$ (dry weight) observed in the parental strain). Thus, the kinetics of phenylalanine uptake in Y294 $\Delta$ agpl did not deviate significantly from the kinetics of a single transport system owing to the presence of two permeases of similar affinity: Bap2p and the unknown 
phenylalanine permease $\left(K_{T}=67 \mu \mathrm{M}\right.$ and $J_{\max }=$ $0.21 \mu \mathrm{mol} / \mathrm{min} / \mathrm{g}$ of dry weight). This activity is not due to residual Gaplp activity under our experimental conditions (Table 2).

To further characterize the specificity of the unknown permease $\left(K_{T}=67 \mu \mathrm{M}\right)$ we measured uptake of $20 \mu \mathrm{M} \mathrm{L}$ $\left[{ }^{14} \mathrm{C}\right]$ - phenylalanine uptake in the presence of various amino acids at $200 \mu \mathrm{M}$ of concentration in Y294 $\Delta$ bap 2 strain grown in MGln medium. Valine, tryptophan, tyrosine, isoleucine, and leucine inhibited the phenylalanine transport 30 to $50 \%$.

Regenberg et al. [14] showed that in the strain ssylgap1 the overexpression of the BAP3 gene, but not the TAT2 gene (under control of the constitutive TPIl promoter), caused an increase in the transport of branched-chain amino acids. The Bap3p and Tat $2 p$ permeases are also capable of transporting phenylalanine, tyrosine, and tryptophan. These results suggest that $B A P 3$ may encode the other unidentified phenylalanine permease with a $\mathrm{km}$ of $67 \mu \mathrm{M}$.

Since Agplp shows a high level of low-affinity phenylalanine uptake activity in strain Y294 $\Delta$ gap1 (Figure 1(d)), the kinetics of this permease was studied in both MP and MA

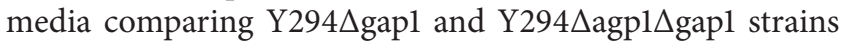
(Table 6). In MP medium the removal of Agplp activity led to the loss of the major part of the uptake capability and the residual uptake was linear with substrate concentration. The apparent $K_{T}$ value of Agplp was found to be $0.75 \mathrm{mM}$ and the $J_{\max }$ value was $14.3 \mu \mathrm{mol} / \mathrm{min}$ per g. of dry weight. With ammonium ion as nitrogen source, the removal of Agplp activity (strain Y294 $\Delta$ agp1 $\Delta$ gapl) also affects the total phenylalanine uptake capability, but to a lesser degree. At least two components are involved in phenylalanine uptake in Y294 agp1 $\Delta$ gap1 strain, as shown by the Eadie-Hofstee plot. The kinetic analysis indicates the presence of a nonsaturable component and one transport system of high-affinity with $K_{T}$ of $39 \mu \mathrm{M}$ and $J_{\max }$ of $0.60 \mu \mathrm{mol} / \mathrm{min}$ per g. of dry weight. No significant difference was seen between the Y294 $\Delta$ bap2 $\Delta$ gap1 and Y294 $\Delta$ gap1 strains in either medium (data not shown).

\section{Discussion}

The experiments in this study indicate that the transport of L-phenylalanine by Saccharomyces cerevisiae is catalyzed primarily by three permeases, Gap1, Bap2p, and Agplp. The relative contribution of these permeases depends upon the type of nitrogen source, the concentration of phenylalanine, and the presence or absence of disruption mutations in one or more of the genes encoding the three permeases. Uptake values for phenylalanine are measured at a $\mathrm{pH}$ of 4.5 units. We consider that this condition is similar to the $\mathrm{pH}$ value of the growth medium in which amino acids uptake is required for cell growth. This does not imply that the relative participation of the different transport systems on phenylalanine uptake could not vary according to changes in $\mathrm{pH}$ values.

Phenylalanine transport in wild type cells grown on medium containing proline as sole nitrogen source (MP medium) is catalyzed by Gaplp, as expected [11, 27]. When ammonium ion is used as nitrogen source, (MA medium) Gaplp activity is absent while phenylalanine uptake remains almost the same. The major permease responsible for highaffinity phenylalanine transport is Bap2p, rather than the broad-specificity permease, Agplp (Figure 1). Disruption of $B A P 2$ results in a dramatic loss of phenylalanine transport activity, whereas disruption of $A G P 1$ has no effect. At relatively high phenylalanine concentration $(1.0 \mathrm{mM})$ a significant fraction of phenylalanine transport involves two more permeases, one of these has intermediate affinity with an apparent $K_{T}$ of $67 \mu \mathrm{M}$ (Table 6). The permease responsible for this activity has not been identified but is not Agplp (Figure 1(b), Table 6). In addition, GAP1 disruption in a bap2 background causes resistance to the phenylalanine analogue $\beta$-(2-thienyl) alanine.

Participation of Agplp in phenylalanine transport is also demonstrated by the growth defect observed when cells are grown on minimal medium containing $1.0 \mathrm{mM}$ phenylalanine as sole nitrogen source (data not shown). It may be noted that although GAP1 was functional in this experiment it was not the only permease involved in phenylalanine uptake. This result extends the experiments of Iraqui et al. [13] who found that disruption of both GAP1 and $A G P 1$ abolished growth on a similar medium.

It is important to emphasize that in our experiments, except for those in Table 1, $0.23 \mathrm{mM}$ leucine was present in all growth media. This ensured that transcription of both AGP1 and BAP2 would be fully activated by SPS-dependent signaling [21].

One of the surprising conclusions which can be drawn from our results is that inactivation/repression of GAP1 permease is not equivalent to GAP1 disruption. The difference is particularly striking when the effects of $A G P 1$ disruption on phenylalanine transport in MA-grown wild type cells and in agplgap1 cells are compared. AGP1 disruption alone caused no decrease in either high- or low-affinity phenylalanine uptake relative to wild type (Figures $1(\mathrm{a})$ and $1(\mathrm{~b})$ ). In contrast, $A G P 1$ disruption caused dramatic decreases in uptake in gapl cells (Figures 1(c) and 1(d)). The GAP1 disruption in MPgrown cells increases low-affinity phenylalanine uptake more than eightfold (Figures 1(b) and 1(d)). Most (76\%) of this high uptake is lost upon introduction of the agpl mutation into the genome. Similarly, $83 \%$ of high-affinity phenylalanine uptake can be attributed to Agplp (Figure 1(c)). Apparently the presence of Gaplp protein in MP-grown wild type cells limits production of active Agplp. Perhaps inactivated Gaplp plays a similar role in wild type cells grown in MA medium.

Y294 gap1 cells grown in MA medium exhibit some loss of low-affinity phenylalanine transport in response to disruptions in either BAP2 or AGP1. These responses may be additive (Figure 1(d)). In this case both Agplp and Bap2p are contributing to low-affinity phenylalanine transport simultaneously. The competitive inhibition studies detailed in Tables 3,4 , and 5 reveal some surprising dynamic relationships between the three principal permeases that can catalyze phenylalanine uptake. These experiments provide an alternative way of identifying which permeases are transporting phenylalanine in different mutants grown in different media. Table 3 shows that for cells grown in MP medium, disruption of $B A P 2$ increases inhibition by citrulline, indicating a greater contribution by Gaplp in high-affinity phenylalanine uptake. 
As expected, trifluoroleucine inhibition is decreased in the bap2 strain. Taken together, the results indicate that Bap2p does in fact contribute to high-affinity phenylalanine uptake in MP-grown cells.

As noted above (Figure 1(d)), disruption of GAP1 causes a major change in the behavior of Agplp, but little or no change in Bap2p (Table 5). Disruption of AGP1 in MP-grown gap1 cells almost eliminates sensitivity of phenylalanine uptake to the three amino acids tested, and uninhibited transport was severely limited. In contrast, disruption of BAP2 had no appreciable effect.

Although the agplgap1 strain retains the BAP2 gene, apparently little or no Bap2p is expressed in MP-grown cells, since competition between high-affinity phenylalanine uptake and leucine is barely detectable (Table 5). Moreover, high-affinity phenylalanine uptake in the absence of inhibitors is very low. In contrast, when the cells are grown in MA medium, high-affinity phenylalanine uptake more than doubles, and inhibition by leucine is restored. That reflects that BAP2 expression is supported by the strong competition of trifluoroleucine and $\beta$-(2-thienyl) alanine.

The kinetic studies reveal another surprising effect; namely, at high phenylalanine concentration in MP-grown cells, a significant level of the phenylalanine transport appears to either be nonsaturable or have an extremely low affinity (Table 6). This component does not appear in MA-grown cells of wild type, or in mutants with single disruptions in $B A P 2$ or AGP1 (Table 6). Here again, the disruption of GAP1 has very different effects on phenylalanine transport systems than repression and inactivation of GAP1 by ammonium ion. As we found in this work, there is an additional phenylalanine transporter, it remains for future studies to establish the nature of that system. A plausible approach would be to study the transport of phenylalanine in gap1, agp1, and bap2 triple mutants.

\section{Conflict of Interests}

The authors declare that they have no conflict of interests regarding the publication of this paper.

\section{Acknowledgment}

The authors dedicate this paper to the memory of Professor Dr. James R. Mattoon (1930-2010).

\section{References}

[1] B. André, "An overview of membrane transport proteins in Saccharomyces cerevisiae," Yeast, vol. 11, no. 16, pp. 1575-1611, 1995.

[2] J. Horák, "Yeast nutrient transporters," Biochim Biophys Acta, vol. 1331, pp. 41-79, 1997.

[3] B. Nelissen, R. de Wachter, and A. Goffeau, "Classification of all putative permeases and other membrane plurispanners of the major facilitator superfamily encoded by the complete genome of Saccharomyces cerevisiae," FEMS Microbiology Reviews, vol. 21, no. 2, pp. 113-134, 1997.
[4] H. Forsberg and P. O. Ljungdahl, "Sensors of extracellular nutrients in Saccharomyces cerevisiae," Current Genetics, vol. 40, no. 2, pp. 91-109, 2001.

[5] J.-C. Jauniaux, M. Vandenbol, S. Vissers, K. Broman, and M. Grenson, "Nitrogen catabolite regulation of proline permease in Saccharomyces cerevisiae. Cloning of the PUT4 gene and study of PUT4 RNA levels in wild-type and mutant strains," European Journal of Biochemistry, vol. 164, no. 3, pp. 601-606, 1987.

[6] J.-C. Jauniaux and M. Grenson, "GAP1, the general amino acid permease gene of Saccharomyces cerevisiae. Nucleotide sequence, protein similarity with the other bakers yeast amino acid permeases, and nitrogen catabolite repression," European Journal of Biochemistry, vol. 190, no. 1, pp. 39-44, 1990.

[7] M. Grenson, "Amino acid transporters in yeast: structure, function and regulation," in Molecular Aspects of Transport Proteins, J. J. H. H. M. de Pont, Ed., pp. 219-245, Elsevier, 1992.

[8] B. Magasanik, "Regulation of nitrogen utilization," in Molecular and Cellular Biology of the Yeast Saccharomyces. Gene Expression, E. W. Jones, J. R. Pringle, and J. R. Broach, Eds., pp. 283317, Cold Spring Harbor Laboratory Press, Cold Spring Harbor, NY, USA, 1992.

[9] M. Hanscho, D. E. Ruckerbauer, N. Chauhan et al., "Nutritional requirements of the BY series of Saccharomyces cerevisiae strains for optimum growth," FEMS Yeast Research, vol. 12, pp. 796-808, 2012.

[10] L. Crépin, T. Nidelet, I. Sanchez, S. Dequin, and C. Camarasa, "Sequential use of nitrogen compounds by Saccharomyces cerevisiae during wine fermentation: a model based on kinetic and regulation characteristics of nitrogen permeases," Applied and Environmental Microbiology, vol. 78, pp. 8102-8111, 2012.

[11] R. L. Greasham and A. G. Moat, "Amino acid transport in a polyaromatic amino acid auxotroph of Saccharomyces cerevisiae," Journal of Bacteriology, vol. 115, no. 3, pp. 975-981, 1973.

[12] H. Klasson, G. R. Fink, and P. O. Ljungdahl, "Ssylp and Ptr3p are plasma membrane components of a yeast system that senses extracellular amino acids," Molecular and Cellular Biology, vol. 19, no. 8, pp. 5405-5416, 1999.

[13] I. Iraqui, S. Vissers, F. Bernard et al., "Amino acid signaling in Saccharomyces cerevisiae: a permease-like sensor of external amino acids and F-box protein Grrlp are required for transcriptional induction of the AGP1 gene, which encodes a broad-specificity amino acid permease," Molecular and Cellular Biology, vol. 19, no. 2, pp. 989-1001, 1999.

[14] B. Regenberg, L. Düring-Olsen, M. C. Kielland-Brandt, and S. Holmberg, "Substrate specificity and gene expression of the amino acid permeases in Saccharomyces cerevisiae," Current Genetics, vol. 36, no. 6, pp. 317-328, 1999.

[15] M. Grauslund, T. Didion, M. C. Kielland-Brandt, and H. A. Andersen, " $B A P 2$, a gene encoding a permease for branchedchain amino acids in Saccharomyces cerevisiae," Biochimica et Biophysica Acta, vol. 1269, no. 3, pp. 275-280, 1995.

[16] M. Grenson, C. Hou, and M. Crabeel, "Multiplicity of the amino acid permeases in Saccharomyces cerevisiae. IV. Evidence for a general amino acid permease," Journal of Bacteriology, vol. 103, no. 3, pp. 770-777, 1970.

[17] J. Schreve and J. M. Garrett, "The branched-chain amino acid permease gene of Saccharomyces cerevisiae, BAP2, encodes the high-affinity leucine permease (S1)," Yeast, vol. 13, pp. 435-439, 1997. 
[18] J. L. Schreve, J. K. Sin, and J. M. Garrett, “The Saccharomyces cerevisiae YCC5 (YCL025c) gene encodes an amino acid permease, Agp1, which transports asparagine and glutamine," Journal of Bacteriology, vol. 180, no. 9, pp. 2556-2559, 1998.

[19] X. Zhu, J. Garrett, J. Schreve, and T. Michaeli, "GNP1, the high affinity glutamine permease of S. cerevisiae," Current Genetics, vol. 30, no. 2, pp. 107-114, 1996.

[20] T. Didion, M. Grauslund, M. C. Kielland-Brandt, and H. A. Andersen, "Amino acids induce expression of BAP2, a branched-chain amino acid permease gene in Saccharomyces cerevisiae," Journal of Bacteriology, vol. 178, no. 7, pp. 2025-2029, 1996.

[21] T. Didion, B. Regenberg, M. U. Jørgensen, M. C. KiellandBrandt, and H. A. Andersen, "The permease homologue Ssylp controls the expression of amino acid and peptide transporter genes in Saccharomyces cerevisiae," Molecular Microbiology, vol. 27, no. 3, pp. 643-650, 1998.

[22] M. de Boer, J.-P. Bebelman, P. M. Gonçalves, J. Maat, H. van Heerikhuizen, and R. J. Planta, "Regulation of expression of the amino acid transporter gene BAP3 in Saccharomyces cerevisiae," Molecular Microbiology, vol. 30, no. 3, pp. 603-613, 1998.

[23] A. Schmidt, M. N. Hall, and A. Koller, "Two FK506 resistanceconferring genes in Saccharomyces cerevisiae, TAT1 and TAT2, encode amino acid permeases mediating tyrosine and tryptophan uptake," Molecular and Cellular Biology, vol. 14, no. 10, pp. 6597-6606, 1994.

[24] N. Kanda and F. Abe, "Structural and functional implications of the yeast high-affinity tryptophan permease Tat2," Biochemistry, vol. 52, pp. 4296-4307, 2013.

[25] H. Forsberg, C. F. Gilstring, A. Zargari, P. Martínez, and P. O. Ljungdahl, "The role of the yeast plasma membrane SPS nutrient sensor in the metabolic response to extracellular amino acids," Molecular Microbiology, vol. 42, no. 1, pp. 215-228, 2001.

[26] M. S. Chianelli, C. A. Stella, D. A. Sáenz, E. H. Ramos, N. Kotliar, and J. R. Mattoon, "Isolation of a trifluoroleucine-resistant mutant of Saccharomyces cerevisiae deficient in both highand low-affinity L-leucine transport," Cellular and Molecular Biology, vol. 42, no. 6, pp. 847-857, 1996.

[27] T. G. Cooper, "Transport in Saccharomyces cerevisiae," in The Molecular Biology of the Yeast Saccharomyces: Metabolism and Gene Expression, N. Strathern, E. W. Jones, and J. R. Broach, Eds., pp. 399-462, Cold Spring Harbor Laboratory Press, Cold Spring Harbor, NY, USA, 1982. 

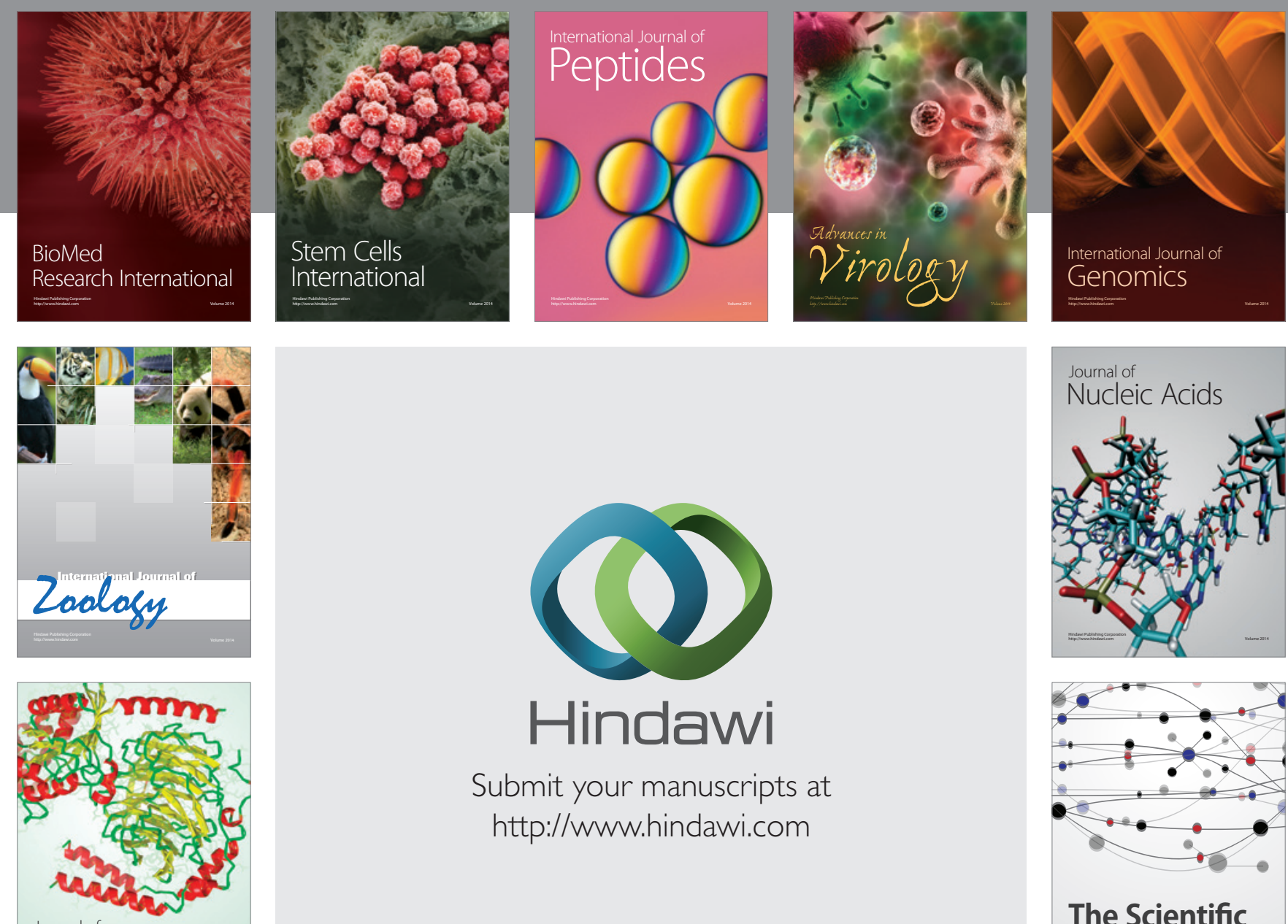

Submit your manuscripts at

http://www.hindawi.com

Journal of
Signal Transduction
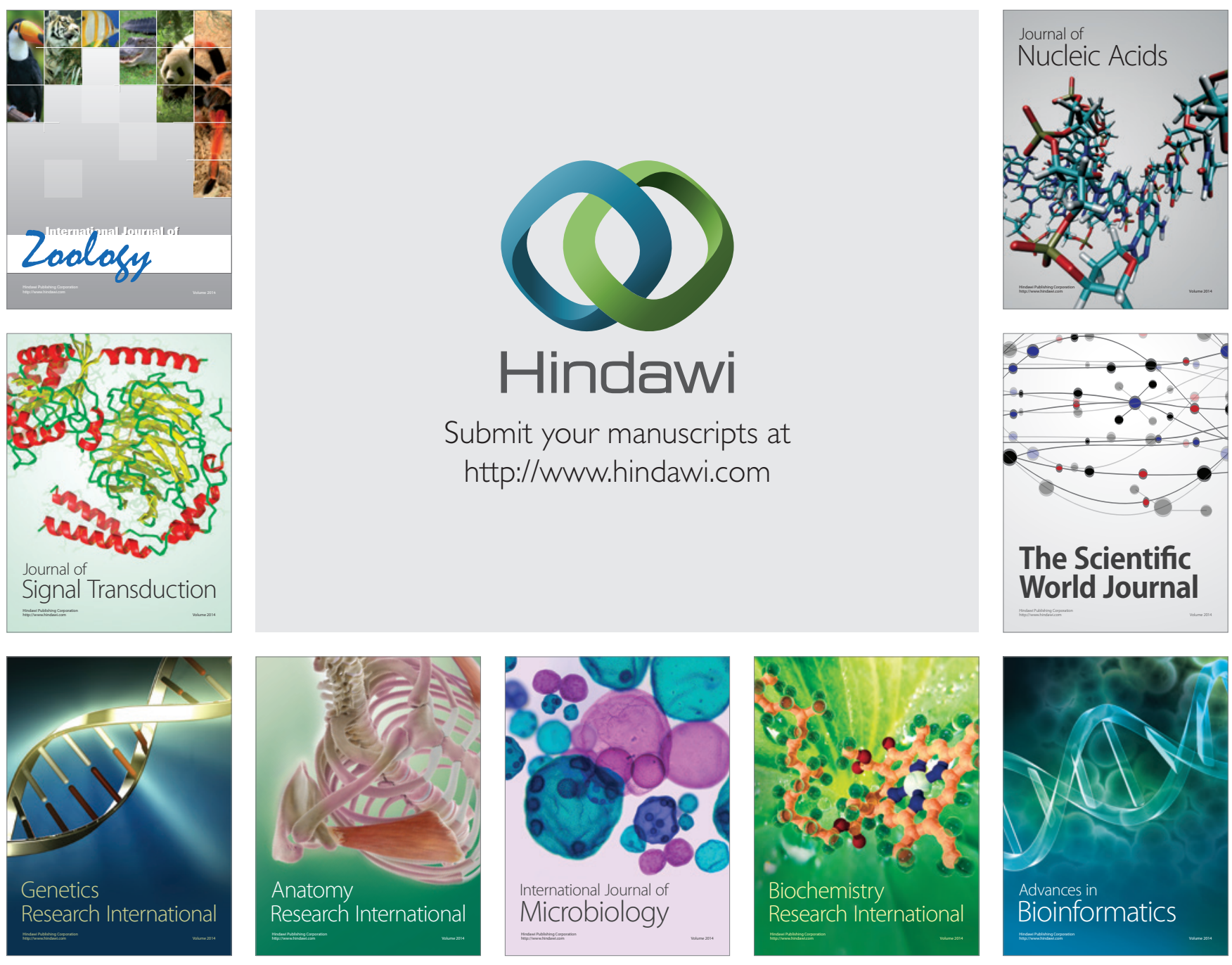

The Scientific World Journal
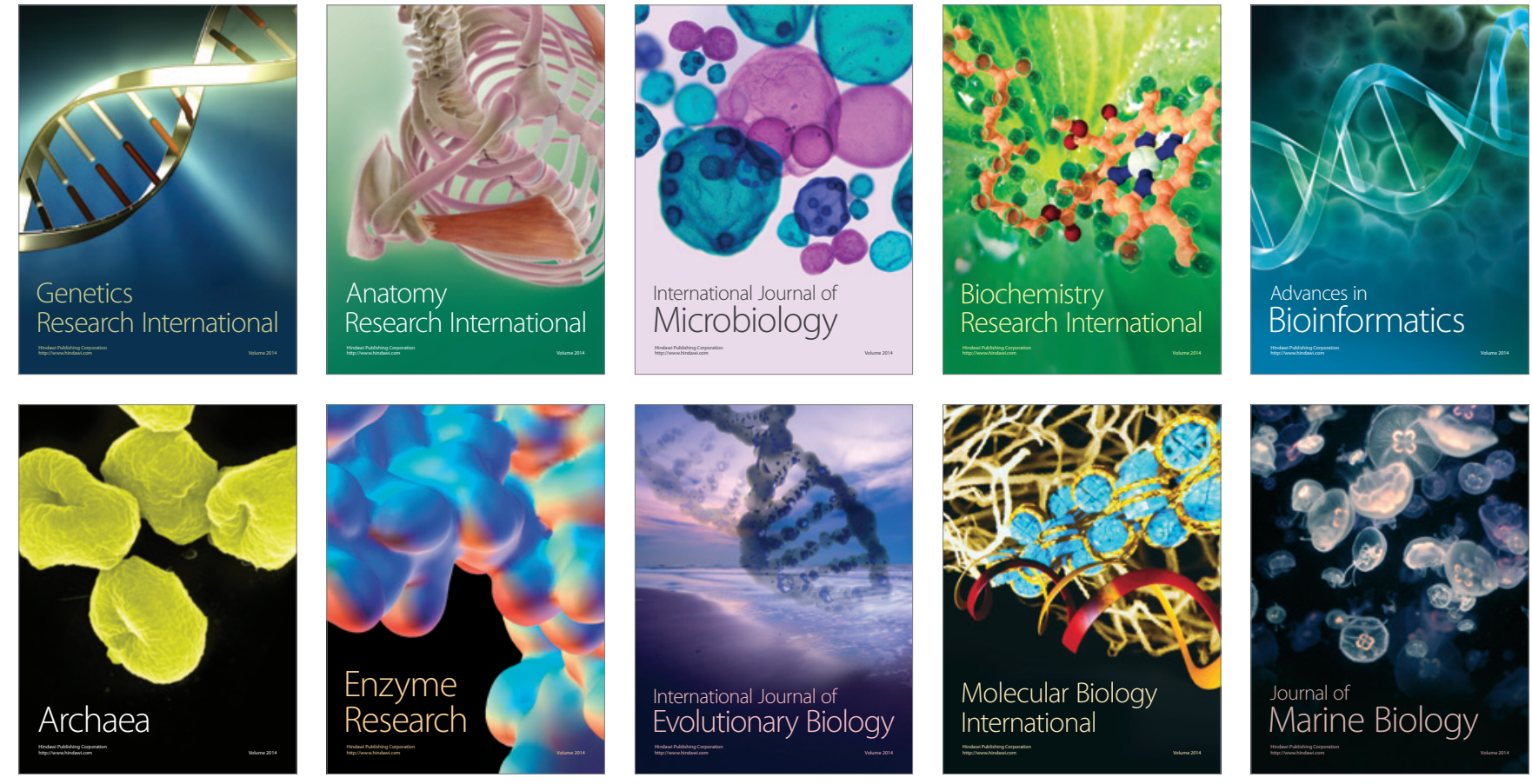